\title{
Autocompensation of Reactive Power in Electric Networks
}

\author{
Valentine D. Pavlov* \\ Vladimir Electromechanical Plant \\ Vladimir, Russian Federation
}

Received 09.08.2021, received in revised form 12.08.2021, accepted 30.08.2021

\begin{abstract}
The aim of this work is to assess the influence of a cascade of transformers and shunt reactors on the reactive power in the network at a symmetrical load. It has been established that any phase is a source of reactive power for the other two phases. Conversely, any two phases are the source of the third phase reactive power. Thus, with a symmetrical load, self-compensation of reactive power occurs. A cascade of transformers is represented by their simplified equivalent circuits (excluding scattering). If the transformers are identical, then the reactive power flow from the load is divided between them into equal parts. Therefore, the reactive power flow in the outer section is three times less than in the inner section. In accordance with this, in a real network, with an increase in the number of transformer substations and shunt reactors, the reactive power flow significantly decreases with distance from the load.
\end{abstract}

Keywords: reactive power, self-compensation, symmetrical load, transformer cascade.

\section{Автокомпенсация реактивной мощности}

\section{в электрических сетях}

\author{
В.Д. Павлов \\ Владимирский электромеханический завод \\ Российская Федерация, Владимир
}

Аннотация. Целью работы является оценка влияния каскада трансформаторов и шунтирующих реакторов на реактивную мощность в сети при симметричной нагрузке. Установлено, что любая фаза является источником реактивной мощности двух других фаз. И наоборот, любые две фазы являются источником реактивной мощности третьей фазы. Таким образом, при симметричной

(C) Siberian Federal University. All rights reserved

This work is licensed under a Creative Commons Attribution-Non Commercial 4.0 International License (CC BY-NC 4.0).

* Corresponding author E-mail address: pavlov.val.75@mail.ru 
нагрузке происходит самокомпенсация реактивной мощности. Каскад трансформаторов представлен их упрощенными схемами замещения (без учета рассеяний). Если трансформаторы идентичны, то поток реактивной мощности от нагрузки делится между ними на равные части. Поэтому поток реактивной мощности на внешнем участке в три раза меньше, чем на внутреннем участке. В соответствии с этим в реальной сети с ростом числа трансформаторных подстанций и шунтирующих реакторов поток реактивной мощности по мере удаления от нагрузки существенно уменьшается.

Ключевые слова: реактивная мощность, самокомпенсация, симметричная нагрузка, каскад трансформаторов.

Цитирование: Павлов, В. Д. Автокомпенсация реактивной мощности в электрических сетях / В. Д. Павлов // Журн. Сиб. федер. ун-та. Техника и технологии, 2021, 14(6). С. 684-688. DOI: 10.17516/1999-494X-0342

Реактивная мощность в сети - нежелательное явление $[1,2]$. Ее циркуляция по проводам вызывает тепловые потери в объеме примерно $10 \%$ ее величины. Для ее снижения используют дорогостоящие статические и динамические компенсаторы.

Цель работы - оценка влияния каскада трансформаторов и шунтирующих реакторов на реактивную мощность в сети при симметричной нагрузке.

В настоящей работе применяются электротехнические расчеты.

\section{Самокомпенсация реактивной мощности при симметричной нагрузке}

На рис. 1 представлена существенно идеализированная схема подключения вторичных обмоток трансформатора к симметричной индуктивной нагрузке [3]. Сопротивления рассеяния не показаны. Активные составляющие сопротивлений не рассматриваются. Вращающееся магнитное поле создается первичными обмотками (не показаны). Реактивная мощность в сеть не передается (гипотетически).

В части вращающегося магнитного поля и опосредованного взаимодействия его с нагрузкой схема неотличима от рассмотренной в [4], в которой вместо трансформатора использована синхронная явнополюсная машина и в которой источником возбуждения колебаний тока (индуктивного) в любой фазе служат другие (другая) фазы (фаза) нагрузки.

Подобное явление самокомпенсации реактивной мощности известно и в механических системах [5]

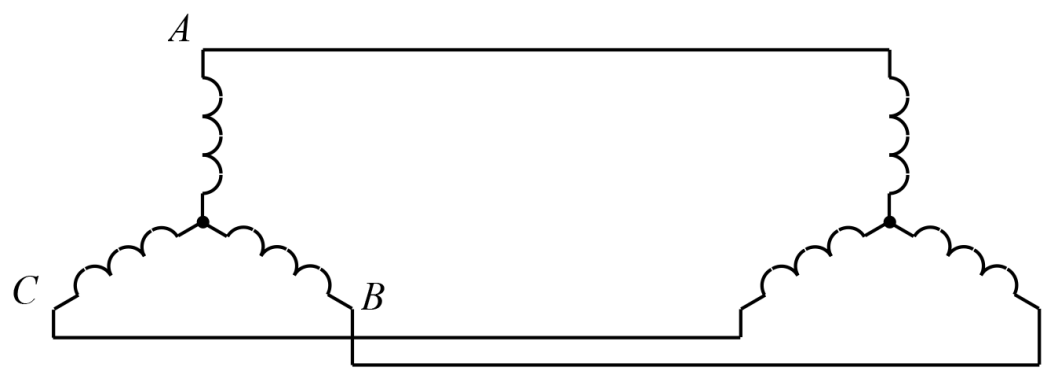

Рис. 1. Самокомпенсация реактивной мощности

Fig. 1. Self-compensation of reactive power 
Мгновенные токи, напряжения и мощности (реактивные) фаз, соответственно, равны

$$
\begin{aligned}
& i_{A}=I \sin \omega t, \\
& u_{A}=I \omega L \cos \omega t, \\
& q_{A}=I^{2} \omega L \sin \omega t \cos \omega t=\frac{1}{2} I \omega L \sin 2 \omega t, \\
& i_{B}=I \sin \left(\omega t-\frac{2 \pi}{3}\right), \\
& u_{B}=I \omega L \cos \left(\omega t-\frac{2 \pi}{3}\right), \\
& q_{B}=\frac{1}{2} I^{2} \omega L \sin \left(2 \omega t-\frac{4 \pi}{3}\right), \\
& i_{C}=I \sin \left(\omega t+\frac{2 \pi}{3}\right), \\
& u_{C}=I \omega L \cos \left(\omega t+\frac{2 \pi}{3}\right), \\
& q_{C}=\frac{1}{2} I^{2} \omega L \sin \left(2 \omega t+\frac{4 \pi}{3}\right) .
\end{aligned}
$$

Здесь $L$ - суммарная индуктивность фазы трансформатора (включая рассеяние) и нагрузки.

Сумма мощностей фаз В и С равна

$$
\begin{aligned}
& q_{B C}=q_{B}+q_{C}=\frac{1}{2} I \omega L \sin \left(2 \omega t-\frac{4 \pi}{3}\right)+\frac{1}{2} I \omega L \sin \left(2 \omega t+\frac{4 \pi}{3}\right)= \\
& =2 \frac{1}{2} I \omega L \sin \frac{2 \omega t-4 \pi / 3+2 \omega t+4 \pi / 3}{2} \cos \frac{2 \omega t-4 \pi / 3-2 \omega t-4 \pi / 3}{2}= \\
& =2 \frac{1}{2} I \omega L \sin 2 \omega t \cos \left(-\frac{4 \pi}{3}\right)=-\frac{1}{2} I \omega L \sin 2 \omega t .
\end{aligned}
$$

Эта величина противоположна мощности фазы $A$, т. е.

$$
q_{B}+q_{C}=-q_{A} .
$$

Это означает, что при оговоренных условиях любая фаза является источником реактивной мощности двух других фаз.

И наоборот, любые две фазы являются источником реактивной мощности третьей фазы.

Таким образом, для данной схемы при симметричной нагрузке происходит самокомпенсация реактивной мощности. 


\section{Замечание о мощностях фаз}

Для того чтобы последняя формула не создавала впечатление, что мощность в любом случае удовлетворяет аналогу первого закона Кирхгофа, ниже приводятся выражения для мгновенных активных мощностей фаз и их соотношение.

$$
\begin{aligned}
& p_{A}=I^{2} \omega L \cos ^{2} \omega t=I^{2} \omega L \frac{1+\cos 2 \omega t}{2}=\frac{1}{2} I^{2} \omega L+\frac{1}{2} I^{2} \omega L \cos 2 \omega t \\
& p_{B}=\frac{1}{2} I^{2} \omega L+\frac{1}{2} I^{2} \omega L \cos \left(2 \omega t-\frac{4 \pi}{3}\right) \\
& p_{C}=\frac{1}{2} I^{2} \omega L+\frac{1}{2} I^{2} \omega L \cos \left(2 \omega t+\frac{4 \pi}{3}\right) \\
& p_{B}+p_{C}=\frac{1}{2} I^{2} \omega L+\frac{1}{2} I^{2} \omega L \cos \left(2 \omega t-\frac{4 \pi}{3}\right)+\frac{1}{2} I^{2} \omega L+\frac{1}{2} I^{2} \omega L \cos \left(2 \omega t+\frac{4 \pi}{3}\right)= \\
& =I^{2} \omega L-\frac{1}{2} I^{2} \omega L \frac{1}{2} \cos 2 \omega t-\frac{1}{2} I^{2} \omega L \frac{1}{2} \cos 2 \omega t=I^{2} \omega L-\frac{1}{2} I^{2} \omega L \cos 2 \omega t \\
& p_{B}+p_{C} \neq-p_{A}
\end{aligned}
$$

\section{Потоки реактивной мощности в каскаде трансформаторов}

Каскад трансформаторов на рис. 2 представлен их упрощенными схемами замещения (без учета рассеяний). Активные сопротивления не рассматриваются.

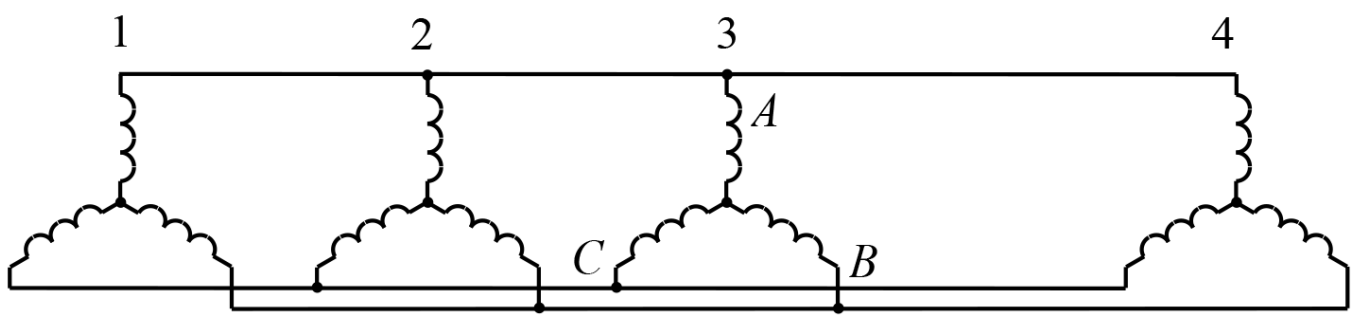

Рис. 2. Каскад трансформаторов

Fig. 2. Transformer cascade

Идеализация этой схемы меньше, чем первой, поскольку реактивная мощность второго и третьего трансформаторов передается в первичную обмотку.

Если трансформаторы идентичны, то поток реактивной мощности от нагрузки делится между ними на равные части. Поэтому поток реактивной мощности на участке 1-2 в три раза меньше, чем на участке 3-4.

В соответствии с этим в реальной сети с ростом числа трансформаторных подстанций и шунтирующих реакторов поток реактивной мощности по мере удаления от нагрузки существенно уменьшается за счет взаимной компенсации реактивной мощности фаз.

$$
-687-
$$




\section{Список литературы / References}

[1] Кузьмин С.В., Завалов А.А., Кузьмин Р.С., Меньшиков В.А. Повышение эффективности компенсации реактивной мощности на основе устройств пофазной компенсации. Журн. Сиб. федер. ун-та. Техника и технологии, 2020, 13(1), 14-24. DOI: 10.17516/1999-494X-0187 [Kuzmin S. V., Zavalov A. A., Kuzmin R.S., Menshikov V.A. Improving the efficiency of reactive power compensation based on phase compensation devices. J. Sib. Fed. Univ. Eng. \& Technol., 2020, 13(1), 14-24. DOI: 10.17516/1999-494X-0187 (in Russian)]

[2] Шеркунков М.А., Тигунцев С.Г. Метод совместной компенсации реактивной мощности, симметрирования нагрузки и фильтрации токов гармоник несимметричной нелинейной нагрузки, соединенной в треугольник. Журн. Сиб. федер. ун-та. Техника и технологии, 2020, 13(4), 455-461. DOI: 10.17516/1999-494X-0237 [Sherkunkov M. A., Tiguntsev S. G. Method of joint compensation of reactive power, load balancing and filtering of harmonic currents of an asymmetrical non-linear load connected in a delta. J. Sib. Fed. Univ. Eng. \& Technol., 2020, 13(4), 455-461. DOI: 10.17516/1999-494X-0237 (in Russian)]

[3] Romanova V.V., Khromov S. V., Suvorov I.F. Development of procedures for determining the optimal placement of symmetration devices for electrical supply systems $0.4 \mathrm{KV}$ with motor-actuated load. J. Sib. Fed. Univ. Eng. \& Technol., 2018, 11(5), 528-535. DOI: 10.17516/1999-494X-0051

[4] Попов И.П. Свободные гармонические колебания в электрических системах с однородными реактивными элементами. Электричество, 2013, (1), 57-59 [Popov I.P. Free harmonic oscillations in electrical systems with homogeneous reactive elements. Electricity, 2013, (1), 57-59 (in Russian)]

[5] Popov I. P. Free harmonic oscillations in systems with homogeneous elements. Journal of Applied Mathematics and Mechanics, 2012, 76(4), 393-395. DOI: 10.1016/j.jappmathmech.2012.09.005 